\title{
Doses em tomografia computadorizada de crânio: impacto do uso do controle automático de exposição
} Computed tomography of head: impact of the use of automatic exposure control

\author{
Giordana Salvi de Souza ${ }^{1}$, Ana Paula Pastre Froner $^{1}$, Ana Maria Marques da Silva ${ }^{1}$
}

${ }^{1}$ Núcleo de Pesquisa em Imagens Médicas, PUCRS, Porto Alegre, Brasil

\begin{abstract}
Resumo
O uso de sistemas de redução de dose na tomografia computadorizada é particularmente importante para pacientes pediátricos, que possuem uma alta radiossensibilidade, pois estão em fase de crescimento. $O$ objetivo deste estudo foi avaliar o impacto do uso do sistema AEC Care Dose da Siemens na estimativa de dose em exames de tomografia computadorizada de crânio em pacientes pediátricos. Foi realizado um estudo retrospectivo com 199 exames de tomografia computadorizada de crânio de pacientes pediátricos em diferentes faixas etárias, em um tomógrafo Siemens Emotion de 16 canais. Foi possível observar, para todas as faixas etárias, que o uso do sistema AEC Care Dose não apenas reduz o CTDlvol, mas também a amplitude interquartil, reduzindo a dose total na população investigada. Conclui-se que o sistema AEC Care Dose modela a corrente de tubo de acordo com as dimensões do paciente, sendo possível a redução da dose individual e coletiva, sem afetar a qualidade do diagnóstico.
\end{abstract}

Palavras-chave: dosimetria; tomografia computadorizada; controle automático de exposição; Care Dose.

\section{Abstract}

The use of dose reduction systems in computed tomography is particularly important for pediatric patients, who have a high radiosensitivity, since they are in the growing phase. The aim of this study was to evaluate the impact of Siemens AEC Care Dose system use on dose estimation in head computed tomography scans in pediatric patients. A retrospective study was conducted with 199 computed tomography head scans of pediatric patients, divided in different age groups, on a 16-channel Siemens Emotion scanner. It was possible to observe, for all age groups, that the use of AEC Care Dose system, not only reduced CTDI vol, but also the interquartile range, reducing the total dose in the investigated population. Concluding, AEC Care Dose system models the tube current according to the patient's dimensions, reducing individual and collective dose without affecting the diagnostic quality .

Keywords: dosimetry; computed tomography; automatic exposition control; Care Dose.

\section{Introdução}

Existe um consenso na literatura sobre as altas doses que os pacientes pediátricos recebem a partir de um exame de tomografia computadorizada (TC), e que podem resultar em um aumento do risco de carcinogênese induzida por radiação ${ }^{1-3}$.

Pesquisadores vem estudando uma forma mais realística de estimar a dose em TC, considerando o tamanho específico ${ }^{4,5}$ e 0 peso do paciente ${ }^{6}$. Diversos estudos tem demostrado que o uso do controle automático de exposição ou AEC (Automatic Exposition Control) $^{7-9}$ e/ou modificações na tensão do tubo de raios $\mathrm{X}$, pitch ${ }^{10}$ e espessura do corte, podem reduzir a dose no paciente, sem afetar significativamente a qualidade da imagem ${ }^{11}$.

O descritor CTDIvol (Volumetric Computed Tomography Dose Index) é uma estimativa de dose padronizada em exames de TC, que utiliza um simulador cilíndrico padrão de $32 \mathrm{~cm}$ ou $16 \mathrm{~cm}$, dependendo da faixa etária e/ou região do corpo ${ }^{12}$. Tais valores, no entanto, representam apenas uma aproximação da dose absorvida pelo paciente e dependem dos parâmetros selecionados de aquisição, sem considerarem as características antropométricas dos pacientes.
Atualmente, os equipamentos mais modernos de TC possuem sistemas de AEC, que possibilitam a modulação da corrente no tubo de raios $X$, conforme a variação de espessura do paciente. As diferenças de densidade dos tecidos que compõem a região imageada também interferem na qualidade do contraste da imagem. O sistema AEC busca determinar uma fluência de fótons ideal para cada paciente e cada região anatômica analisada ${ }^{13}$. Cada um dos sistemas AEC implementados pelos fabricantes possui especificações diferentes e opera de forma distinta ${ }^{14}$

A fabricante Siemens desenvolveu um sistema AEC denominado Care Dose, que estima a atenuação dos raios $X$ nos planos $x$ e $y$ em cada corte, durante uma rotação. $O$ sistema utiliza tais informações para alimentar um modelo matemático que determina um valor de referência do produto corrente-tempo (mAs) para o próximo corte, controlando a corrente no tubo de raios $X$ para manter um nível adequado de qualidade da imagem, baseado no nível de ruído ${ }^{15}$.

Outros fabricantes desenvolveram diferentes sistemas, como DoseRight, Z-Dom e D-Dom da Philips, que funciona por imagem de referência, e o 
operador seleciona apenas a relação $\mathrm{mAs} /$ pitch para sistema $A E C$ ajustar a modulação da corrente do tubo para alcançar o nível de ruído da imagem de referência. A Toshiba possui o sistema AEC SureExposure 3D e a General Electric possui o Auto+Smart mA, que utilizam índice de ruído (NI) para descrever o nível de ruído em uma imagem com filtro de reconstrução padrão no centro do objeto digitalizado e o operador seleciona o máximo e mínimo do produto corrente-tempo que o AEC pode alcançar e qual o índice de ruído desejado ${ }^{13}$. O trabalho de Reina ${ }^{13}$ estudou o comportamento desses três sistemas AEC e como eles modificam o produto corrente-tempo e a dose com a variação da espessura, utilizando o phantom ImPACT.

O uso de sistemas de redução de dose na TC é particularmente importante para pacientes pediátricos, que possuem uma alta radiossensibilidade, pois estão em fase de crescimento. Autores mostraram que o uso do modulador de dose/corrente em exames de crânio resulta em uma redução de $45 \%$ na dose, sem diferença significativa na qualidade da imagem ${ }^{17,18}$.

O objetivo deste estudo foi avaliar o impacto do uso do sistema AEC Care Dose da Siemens na estimativa de dose em exames de TC de crânio em pacientes pediátricos.

\section{Materiais e Métodos}

Foi realizado um estudo retrospectivo a partir do levantamento de dados entre os anos 2013 a 2015, em um hospital de grande porte, dos exames de TC de crânio realizados em um tomógrafo Siemens Emotion 16 (Siemens Medical Solutions, Erlangen, Germany). Os dados fazem parte de projeto aprovado pelo Comitê de Ética em Pesquisa (CAAE 18924113000005336, parecer 381.090).

Os pacientes pediátricos $(\mathrm{N}=199)$, menores de 15 anos, foram divididos em faixas etárias. A Tabela 1 apresenta $o$ número de exames de pacientes pediátricos analisados, sem e com o uso do sistema AEC Care Dose.

Tabela 1: Número de exames de pacientes pediátricos analisados.

\begin{tabular}{lcccc}
\hline $\begin{array}{c}\mathbf{0}-\mathbf{1} \\
\text { ano }\end{array}$ & $\begin{array}{c}\mathbf{1} \dashv \mathbf{5} \\
\text { anos }\end{array}$ & $\begin{array}{c}\mathbf{5}-\mathbf{1 0} \\
\text { anos }\end{array}$ & $\begin{array}{c}\mathbf{1 0}-\mathbf{1 5} \\
\text { anos }\end{array}$ \\
\hline Sem AEC & 7 & 15 & 19 & 26 \\
Com AEC & 52 & 40 & 34 & 6 \\
\hline
\end{tabular}

Foram coletados os seguintes parâmetros de aquisição das imagens, com e sem 0 uso do sistema AEC Care Dose: tensão do tubo de raios $\mathrm{X}$ $(\mathrm{kVp})$, produto corrente-tempo (mAs) e descritor de dose (CTDlvol).

A distribuição estatística dos parâmetros coletados, com a média, mediana, primeiro e terceiro quartil, além dos valores máximo e mínimo, foi avaliada para as diferentes faixas etárias, sem e com o uso do sistema AEC Care Dose.

\section{Resultados}

A tabela 2 apresenta a média e desvio padrão da tensão no tubo para pacientes pediátricos.
Tabela 2:Média e desvio-padrão da tensão no tubo de raios $\mathrm{X}$, em $\mathrm{kV}$, para exames de pacientes pediátricos.

\begin{tabular}{lcccc} 
& $\begin{array}{c}\mathbf{0}-\mathbf{1} \\
\text { ano }\end{array}$ & $\begin{array}{c}\mathbf{1}-\mathbf{5} \\
\text { anos }\end{array}$ & $\begin{array}{c}\mathbf{5}-\mathbf{1 0} \\
\text { anos }\end{array}$ & $\begin{array}{c}\mathbf{1 0}-15 \\
\text { anos }\end{array}$ \\
\hline Sem AEC & $118 \pm 11$ & $121 \pm 10$ & $127 \pm 8$ & $128 \pm 5$ \\
Com AEC & $109 \pm 4$ & $110 \pm 0$ & $111 \pm 3$ & $110 \pm 0$ \\
\hline
\end{tabular}

A tabela 3 apresenta a média e desvio padrão do produto corrente-tempo para pacientes pediátricos.

Tabela 3:Média e desvio-padrão do produto correntetempo, em mAs, para exames de pacientes pediátricos.

\begin{tabular}{lcccc}
\hline & $\begin{array}{c}\mathbf{0}-\mathbf{1} \\
\text { ano }\end{array}$ & $\begin{array}{c}\mathbf{1}-\mathbf{5} \\
\text { anos }\end{array}$ & $\begin{array}{c}\mathbf{5}-\mathbf{1 0} \\
\text { anos }\end{array}$ & $\begin{array}{c}\mathbf{1 0}-\mathbf{1 5} \\
\text { anos }\end{array}$ \\
\hline Sem AEC & $165 \pm 21$ & $159 \pm 34$ & $189 \pm 21$ & $186 \pm 27$ \\
Com AEC & $89 \pm 14$ & $120 \pm 15$ & $138 \pm 13$ & $145 \pm 9$ \\
\hline
\end{tabular}

A figura 1 mostra a distribuição do CTDl vol $_{\text {sem e }}$ com AEC, para exames de TC de crianças na faixa etária de 0 - 1 ano.

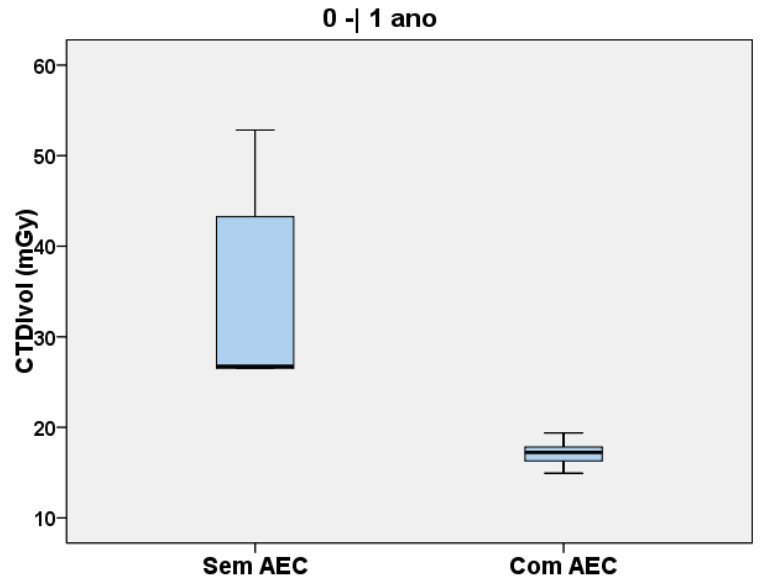

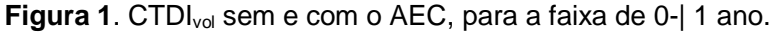

A figura 2 mostra a distribuição do CTDI ${ }_{\text {vol }}$ sem e com AEC, para exames de TC de crianças na faixa etária de 1-| 5 anos.

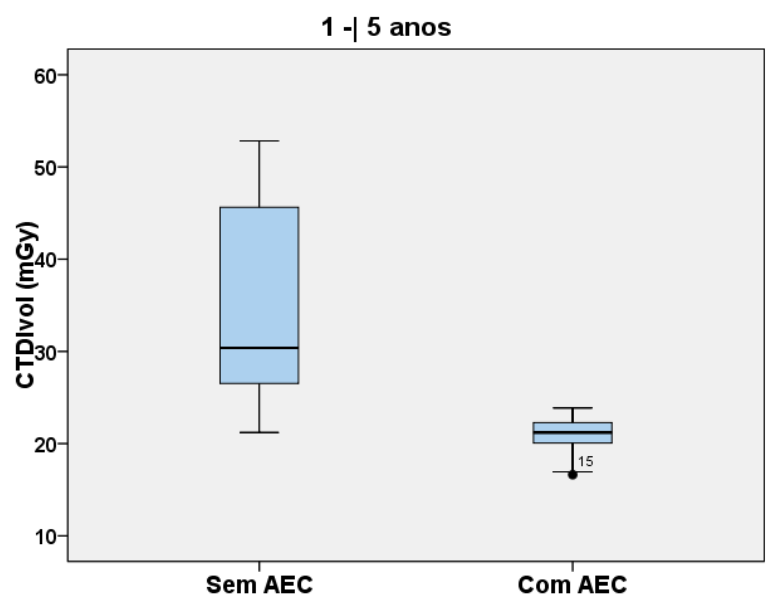

Figura 2. CTDI ${ }_{\text {vol }}$ sem e com o AEC para a faixa de 1-| 5 anos. 
A figura 3 mostra a distribuição do CTDlvol sem e com AEC, para exames de TC de crianças na faixa etária de 5-| 10 anos.

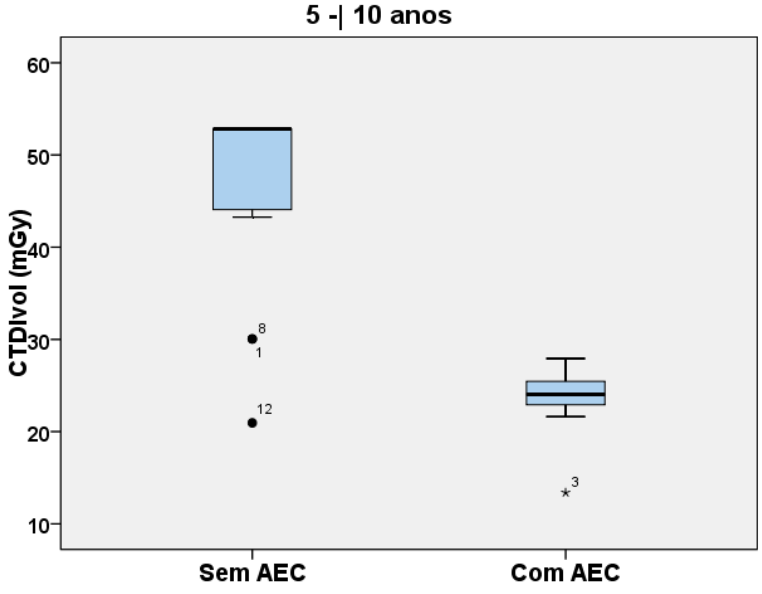

Figura 3. CTDI ${ }_{\text {vol }}$ sem e com o AEC para a faixa de 5-| 10 anos.

A figura 4 mostra a distribuição do CTDlvol sem e com AEC, para exames de TC de crianças na faixa etária de 10-| 15 anos.

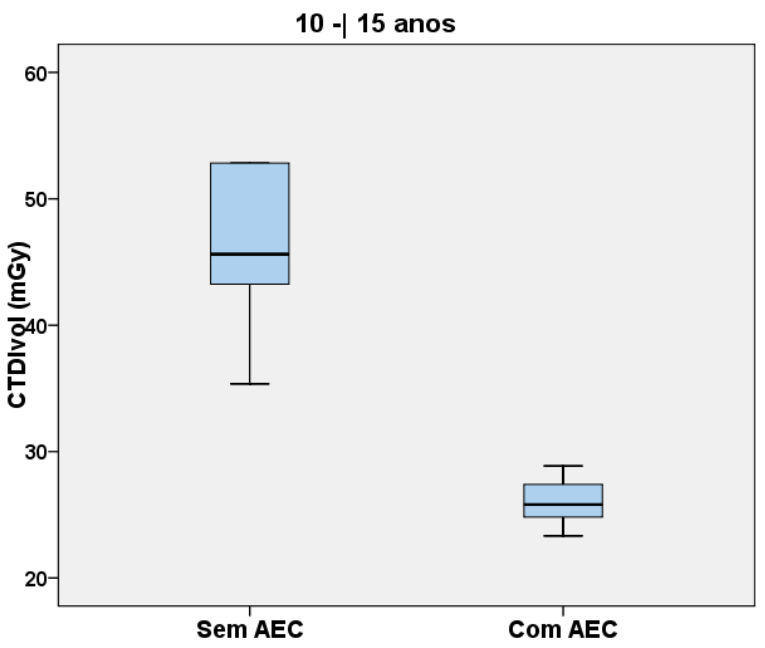

Figura 4. CTDI ${ }_{\text {vol }}$ sem e com o AEC para a faixa de 10-| 15 anos.

\section{Discussão}

A investigação dos protocolos e doses em exames de TC em uma instituição é um trabalho exaustivo, em função do grande número de dados a serem avaliados. Neste estudo, a partir do levantamento retrospectivo de exames de pacientes pediátricos, foi possível observar uma redução da tensão no tubo de raios $X$, quando é utilizado o sistema AEC Care Dose, de 8 a 14\%. A redução do produto corrente-tempo chega a ter uma redução de 22 a 46\%. Estas reduções podem ser diretamente associadas a um aumento da vida útil do tubo de raios $X$, em função do menor aquecimento.

Quando o sistema AEC Care Dose é utilizado, ocorre não apenas uma redução estatisticamente

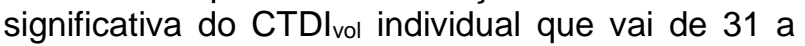
$55 \%$, mas também uma redução da amplitude interquartil da população investigada, para todas as faixas etárias. A maior redução do CTDI $_{\text {vol }}$ ocorre para a faixa etária de 0 a 1 ano, onde o uso do AEC produz uma diminuição de $55 \%$ da dose. Assim apesar de observarmos a presença de alguns outliers, principalmente na faixa etária dos 5 aos 10 anos, observa-se uma redução na dose total na população cujas imagens foram adquiridas com 0 uso do sistema AEC.

Estudos anteriores mostram que uma redução semelhante de dose nos órgãos em pacientes adultos utilizando o sistema AEC, chegando a $35 \%$, quando comparado com o uso de uma corrente fixa ${ }^{16}$. A otimização de outros parâmetros, como a utilização de um pitch maior, possibilitou reduções de dose de $44 \%$ utilizando o AEC para pacientes adultos $^{8}$.

A alta dose observada pela média do CTDI $_{\text {vol }}$ dos pacientes pediátricos produzida pelos equipamentos de TC sem o uso do sistema AEC Care Dose, combinada com a grande variabilidade dos protocolos, vem resultando em exames de algumas crianças com altas doses de radiação. Este resultado mostra a importância de implementar estratégias de redução de doses, como o uso do sistema AEC Care Dose.

Deve-se ressaltar a importância do treinamento dos técnicos e tecnólogos de radiologia para a otimização dos protocolos utilizados, não somente utilizando o sistema $\mathrm{AEC}$, mas tentando reduzir outros parâmetros que alteram a dose, sem influenciar significativamente a qualidade da imagem.

\section{Conclusões}

Conclui-se que o sistema AEC Care Dose modela a corrente de tubo de acordo com as dimensões do paciente, produzindo uma redução significativa do valor individual do CTDI vol do paciente e da população sem modificar significativamente 0 diagnóstico. Apesar da implementação do uso do sistema AEC ter reduzido o valor da dose de radiação aos pacientes, maiores investigações são necessárias para identificar os motivos para alguns valores excessivos de dose identificados como outliers no levantamento.

\section{Agradecimentos}

À FAPERGS e PUCRS pelo apoio financeiro, e ao Hospital São Lucas pelo apoio na aquisição dos dados.

\section{Referências}

1. Li, X., Samei, E., Segars, W. P., Sturgeon, G. M., Colsher, J. G. \& Frush, D. P. Patient-specific radiation dose and cancer risk for pediatric chest CT. Radiology 259, 862-874 (2011).

2. Brenner, D. J., Elliston, C. D., Hall, E. J. \& Berdon, W. E. Estimated Risks of RadiationInduced Fatal Cancer from Pediatric CT. AJR 176, 289-296 (2001).

3. Miglioretti, D. L. et al. The use of computed tomography in pediatrics and the associated radiation exposure and estimated cancer risk. JAMA Pediatr. 167, 700-7 (2013). 
4. Moore, B. M., Brady, S. L., Mirro, A. E. \& Kaufman, R. a. Size-specific dose estimate (SSDE) provides a simple method to calculate organ dose for pediatric CT examinations. Medical Physics 41, 71917 (2014).

5. AAPM. Side-Specific Dose Estimates (SSDE) in Pediatric and Adult Body CT Examinations. AAPM: College Park (2011).

6. Miyazaki, O. et al. Estimation of adaptive computed tomography dose index based on body weight in pediatric patients. Radiation Medine 26, 98-103 (2008).

7. Israel, G. M., Cicchiello, L., Brink, J. \& Huda, W. Patient size and radiation exposure in thoracic, pelvic, and abdominal CT examinations performed with automatic exposure control.AJR 195, 1342-1346 (2010).

8. Rampado, O. et al. Effective dose and image quality evaluations of an automatic CT tube current modulation system with an anthropomorphic phantom. European Journal of Radiology 72, 181-187 (2009).

9. Rizzo, S. et al. Comparison of angular and combined automatic tube current modulation techniques with constant tube current CT of the abdomen and pelvis. AJR 186, 673-679 (2006).

10. Hathout, G. M. \& Bhidayasiri, R. Dose and pitch relationship for a particular multislice CT scanner. AJR 8, 953-956 (2005).

11. Al Mahrooqi, K. M. S., Ng, C. K. C. \& Sun, Z. Pediatric Computed Tomography Dose Optimization Strategies: A Literature Review. Journal of Medical Imaging and Radiation Sciences 46, 241-249 (2015).

12. AAPM. The Measurement, Reporting, and Management of Radiation Dose in CT. (2008).

13. Reina, T. R. Avaliação de sistemas de controle automático de exposição em tomografia computadorizada. Dissertação de Mestrado. Universidade de São Paulo (2014).

14.Söderberg, M. \& Gunnarsson, M. Automatic exposure control in computed tomography--an evaluation of systems from different manufacturers. Acta radiology 51, 625-634 (2010).

15.Söderberg, M. \& Gunnarsson, M. The effect of different adaptation strengths on image quality and radiation dose using Siemens Care Dose 4D. Radiation Protection Dosimetry 139, 173179 (2010).

16.Sookpeng, S., Martin, C. J. \& Gentle, D. J. Comparison of different phantom designs for CT scanner automatic tube current modulation system tests. Journal of radiological protection. 33, 735-61 (2013).

17.Santos, J., Foley, S., Paulo, G., McEntee, M. F. \& Rainford, L. The impact of pediatric-specific dose modulation curves on radiation dose and image quality in head computed tomography. Pediatric Radiology. 45, 1814-1822 (2015).

18.Zacharias, C. et al. Pediatric CT: Strategies to lower radiation dose. AJR 200, 950-956 (2013).

\section{Contato:}

Giordana Salvi de Souza

Av. Ipiranga 6681, Pr. 10

90619-900 Porto Alegre - RS

E-mail: giordana.souza@acad.pucrs.br 